guée du CICR, $\mathrm{M}^{\text {me }}$ Claire Frésard, a témoigné de son expérience sur le terrain.

NÉCROLOGIE

\title{
Hommage à Frédéric Siordet
}

M. Frédéric Siordet, ancien membre du CICR et ancien vice-président de l'institution, est décédé le 30 janvier 1991. Avec lui disparaît une des grandes figures du Comité international de la Croix-Rouge.

$M$. Melchior Borsinger, ancien délégué général du CICR pour l'Europe et l'Amérique du Nord, lauréat de la Médaille HenryDunant, fut son ami. Dans l'hommage qu'il rend à ce grand serviteur de la Croix-Rouge, il a su exprimer les sentiments d'admiration et d'affection que partagent aujourd'hui tous ceux qui, au CICR ou au sein du Mouvement international de la Croix-Rouge et du CroissantRouge, avaient reconnu et apprécié les hautes compétences et les qualités de cœur et d'esprit de Frédéric Siordet.

$* * *$

Frédéric Siordet, membre du CICR de 1951 à 1979, et, à plusieurs reprises, pendant cette période, vice-président de l'institution, nous a quittés, le 30 janvier 1991, en laissant derrière lui un immense vide.

C'était un homme d'une grande modestie et incroyablement doué dans des domaines fort divers. Fils de pasteur, il fut juriste. Ses ascendances en firent aussi bien un homme de l'esprit qu'un homme de la raison. Humaniste éclairé, mélomane passionné, maniant une plume aussi alerte que précise, il alliait un grand sens de l'humour «toepfférien» et anglo-saxon à un esprit des plus latins.

Il fut pendant de longues années avocat à Paris et de ce fait conseiller juridique de la Légation de Suisse. Quand celle-ci dut se replier, en 1940, d'abord à Bordeaux puis à Vichy, Frédéric Siordet demeura à Paris pour y exercer des fonctions paraconsulaires sous l'occupation allemande. 
Lorsqu'il quitta Paris en 1943, pour que ses filles puissent poursuivre leurs études en Suisse, Frédéric Siordet fut nommé conseiller du CICR et nous nous liâmes très rapidement $d$ 'amitié.

Ce fut un collègue exquis; courtois et sensible, il cachait sous une grande modestie une profonde connaissance de l'homme, de ses travers, de ses vices et de ses vertus. Son humour, sa bonté et son espièglerie furent, pour ceux qui le connurent et travaillèrent avec lui, d'un grand soutien à une époque difficile et sombre pour toute l'humanité.

La guerre terminée, il fut appelé à participer aux travaux préparatoires qui devaient conduire à une refonte des Conventions de Genève existantes et à l'élaboration notamment de la $\mathrm{IV}^{\mathrm{e}}$ Convention de Genève nouvelle pour la protection des populations civiles en temps de guerre. Ici, son expérience vécue de la guerre et de l'occupation à Paris fut d'une immense utilité.

A partir de 1950, avec Jean Pictet, Claude Pilloud, René-Jean Wilhelm et Jean de Preux, il fut de ceux qui ont le plus largement contribué à la rédaction du Commentaire de ces Conventions. En marge de ses travaux, son humour nous égaya souvent, lorsque par exemple il rédigea un «Résumé des Conventions en lettres P». L'auteur de ces lignes se souvient particulièrement aussi d'une merveilleuse «Plainte pour Pilloud» dont, si sa mémoire est bonne, le commencement se lisait comme suit: «Pauvre petit Pilloud, précairement penché pupitre périlleux profondément perplexé par problèmes propagation principes protection prisonniers pugnaces, pleure pour prononcer paroles propices»!

En outre, Frédéric Siordet représenta le CICR dans de nombreux pays et lors de plusieurs Conférences de la Croix-Rouge; il fut aussi l'auteur de l'ouvrage Inter Arma Caritas, œuvre de réflexion marquante sur les actitivés du CICR pendant la Seconde Guerre mondiale.

Mais Frédéric Siordet n'était, et de loin, pas seulement homme de plume et de cabinet, ce fut aussi un homme d'action. Il effectua pour le CICR de nombreuses et importantes missions, et non des moindres, un tour du monde en 1945 et 1946 où il visita l'ensemble des Sociétés nationales de Croix-Rouge d'Asie, d'Australasie et d'Amérique Centrale et du Nord, dans des conditions qu'on peut imaginer au lendemain immédiat de la guerre.

Une autre mission l'amena pendant l'hiver 1957 de l'Inde au SudEst asiatique, en Chine, en URSS et en Pologne où il fut appelé à exposer aux dirigeants de ces Etats la position du CICR à l'égard du problème, très délicat à l'époque, de la Chine et de Taiwan. En effet, 
le fait que ce fut la Chine du Kuomintang (Tchang-kai-Tchek) qui signa les Conventions de Genève de 1949 et que ce fut la République populaire de Chine (Mao-Tsé-Tung) qui les ratifia, mit en péril la $\mathrm{XIX}^{\mathrm{e}}$ Conférence internationale de la Croix-Rouge à la Nouvelle Delhi. Cette mission n'en fut que davantage nécessaire.

Frédéric Siordet, alors vice-président, fut aussi le porte-parole de la délégation du CICR à la XVIII ${ }^{e}$ Conférence internationale de la CroixRouge à Toronto en 1952, donc en pleine guerre de Corée, où les Etats et les Sociétés nationales des pays marxistes notamment, attaquèrent avec virulence le CICR, alléguant, pour résumer très crûment, qu'il n'était qu'«un instrument de l'impérialisme capitaliste»! Le discours courageux, lucide et objectif que prononça Frédéric Siordet en assemblée plénière de la Conférence restera toujours comme un monument de l'esprit le plus purement Croix-Rouge qui soit.

Autre trait émouvant de l'homme d'action que fut Frédéric Siordet: lorsque Georges Olivet, jeune délégué du CICR plein de promesses, fut tué en 1962 au Katanga, au Zaïre, dans des circonstances particulièrement tragiques et délicates, Frédéric Siordet, alors membre du Comité, se porta volontaire pour ramener sa dépouille.

Frédéric Siordet fut aussi un sportif et un des principaux animateurs du club de quilles du CICR. Nombreuses aussi furent les parties de tennis ou de billard que l'auteur des présentes lignes eut la joie de partager avec lui.

Ainsi nous quitte un très grand serviteur de l'humanité dont la sincérité, l'honnêteté et la transparence que rehaussait sa vive intelligence ont gagné à la cause de la Croix-Rouge de nombreux sceptiques ou même d'adversaires et qui a inspiré toute une génération d'hommes plus jeunes à poursuivre dans cette voie.

Melchior Borsinger 